\title{
Genomic Diversity and Homologous Recombination in Vibrio parahaemolyticus as Revealed by Amplified Fragment Length Polymorphism (AFLP) and Multilocus Sequence Analysis (MLSA)
}

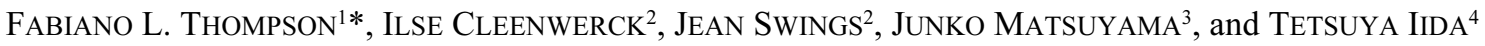 \\ ${ }^{1}$ Department of Genetics, Institute of Biology, Federal University of Rio de Janeiro (UFRJ), CEP 21944-970, \\ RJ, Brazil \\ ${ }^{2}$ Laboratory of Microbiology and BCCM ${ }^{T M} / L M G$ Bacteria Collection, Ghent University, K.L. Ledeganckstraat \\ 35, Ghent 9000, Belgium \\ ${ }^{3}$ Pathogenic Microbes Repository Unit, Research Institute for Microbial Diseases, Osaka University, Suita, \\ Osaka 565-0871, Japan \\ ${ }^{4}$ Laboratory of Genomic Research on Pathogenic Bacteria, International Research Center for Infectious \\ Diseases, Research Institute for Microbial Diseases, Osaka University, Suita, Osaka 565-0871, Japan
}

(Received July 27, 2007-Accepted September 7, 2007)

We examined the genomic diversity of 29 representative Vibrio parahaemolyticus strains based on amplified fragment length polymorphism (AFLP) and multilocus sequence analysis (MLSA). Several strains had indistinguishable AFLP patterns, suggesting high genomic similarity. Recombination detection methods and phylogenetic reconstructions based on the gene sequences of recombination/repair protein (recA), DNA gyrase beta subunit $(\operatorname{gyr} B)$, and phosphoglucomutase $(\mathrm{pgm})$ revealed that recombination has occurred within the species $V$. parahaemolyticus. We suggest that homologous recombination is an important force in the evolution of $V$. parahaemolyticus.

Key words: diversity, homologous recombination, Vibrio parahaemolyticus, AFLP, MLSA

The species Vibrio parahaemolyticus was described in the 1950s during food-borne gastroenteritis outbreaks in Japan that lead to hundreds of cases of diarrhoea associated with the consumption of raw fish. In 1980, V. alginolyticus was proposed as a new species to encompass biotype two of $V$. parahaemolyticus. Phenotypically these two species share many features in common. Of an array of more than 50 tests, only utilization of D-glucosamine, growth at $10 \%$ $\mathrm{NaCl}$, and Voges-Proskauer differ between these species ${ }^{1)}$. $V$. alginolyticus and $V$. parahaemolyticus share $67 \%$ DNADNA hybridization similarity (DDH), and 57\% with the pair $V$. harveyi and $V$. campbelliti ${ }^{521)}$. In spite of the close relatedness between these species, genomic fingerprinting analyses, such as amplified fragment length polymorphism

\footnotetext{
* Corresponding author. E-mail address: fabiano.thompson@biologia.ufrj.br; Tel./Fax: +55-21-25626382.
}

(AFLP) and rep-PCR, and multilocus sequence analysis (MLSA) clearly discriminate the bacterial strains and species $^{5,11,18,20)}$. Currently, researchers are able to identify their isolates through the internet using curated MLSA data of vibrios (http://www.taxvibrio.lncc.br//22,24). Because $V$. parahaemolyticus includes both human and marine animal pathogenic strains ${ }^{7}$, identification at the strain level is crucial for pinpointing the widespread successful clones associated with human and animal health worldwide.

Since 1990s pandemic strains of $V$. parahaemolyticus have emerged from a single clone (serotype O3:K6), associated with hundreds of cases worldwide. Previous pulsed filed gel electrophoresis (PFGE) data indicated that $V$. parahaemolyticus strains have a non-clonal population structure, possibly with recombination between strains giving rise to new epidemic clones ${ }^{9,16,25,26)}$. However, it is not clear yet if recombination occurs in housekeeping genes of $V$. para- 
Table 1. Strain List

\begin{tabular}{|c|c|c|c|c|c|c|c|c|c|}
\hline \multirow{3}{*}{$\begin{array}{l}\text { Strain } \\
\mathrm{vp} 1\end{array}$} & \multirow{3}{*}{$\begin{array}{c}\text { Synonym } \\
\text { RIMD } 2210554\end{array}$} & \multirow{3}{*}{$\begin{array}{r}\text { Source } \\
\text { Patient }\end{array}$} & \multirow{2}{*}{\multicolumn{3}{|c|}{ Place of isolation }} & \multirow{3}{*}{$\begin{array}{c}\begin{array}{c}\text { Year of } \\
\text { Isolation }\end{array} \\
1983\end{array}$} & \multicolumn{2}{|c|}{ PCR } & \multirow{3}{*}{$\begin{array}{l}\text { Serotype } \\
\text { O4:K11 }\end{array}$} \\
\hline & & & & & & & \multirow{2}{*}{$\begin{array}{c}t h \\
-\end{array}$} & \multirow{2}{*}{$\frac{t r h}{+}$} & \\
\hline & & & Japan & Osaka & Osaka aq* & & & & \\
\hline $\mathrm{vp} 2$ & RIMD 2210633 & Patient & Japan & Osaka & Kansai aq & 1996 & + & - & O3:K6 \\
\hline vp3 & RIMD 2210883 & Patient & Japan & Osaka & Osaka aq & 1992 & + & - & O4:K11 \\
\hline vp4 & RIMD 2212470 & Environment & Japan & Toyama & & 2001 & - & - & O5:KUT \\
\hline vp5 & RIMD 2212490 & Environment & Japan & Toyama & & 2001 & - & - & O5:KUT \\
\hline vp6 & RIMD 2210086 & Patient & Japan & Osaka & & 1968 & + & - & $\mathrm{O} 4: \mathrm{K} 12$ \\
\hline vp7 & RIMD 2210536 & Patient & Japan & Osaka & Osaka aq & 1985 & - & + & $\mathrm{O} 3: \mathrm{K} 6$ \\
\hline vp8 & RIMD 2210580 & Environment & Japan & Naha & Naha aq & 1991 & - & - & O5:KUT \\
\hline vp9 & RIMD 2210856 & Patient & Japan & Osaka & Osaka aq & 1991 & + & + & O10:KUT \\
\hline vp10 & RIMD 2211770 & Environment & Thailand & Bangkok & & 1994 & - & - & O10:K61 \\
\hline vp11 & RIMD 2212201 & Environment & Japan & Osaka & & 1999 & - & - & $\mathrm{O} 3: \mathrm{K} 20$ \\
\hline vp12 & RIMD 2212475 & Environment & Japan & Toyama & & 1999 & - & - & O5:KUT \\
\hline vp13 & RIMD 2212673 & Patient & Japan & Osaka & Kansai aq & 2000 & - & + & O1:K56 \\
\hline vp14 & RIMD 21212686 & Patient & Japan & Osaka & Kansai aq & 2001 & + & - & $\mathrm{O} 3: \mathrm{K} 6$ \\
\hline vp15 & RIMD 2212819 & Patient & Japan & Osaka & Kansai aq & 2001 & + & + & O8:K74 \\
\hline vp16 & RIMD 2212924 & Patient & Japan & Osaka & Kansai aq & 2001 & + & - & O4:K68 \\
\hline vp17 & RIMD 22121044 & Patient & Japan & Osaka & Kansai aq & 2001 & + & - & $\mathrm{O} 4: \mathrm{K} 4$ \\
\hline vp18 & RIMD 2210001 & Patient & Japan & Osaka & & 1950 & - & + & $\mathrm{O} 1: \mathrm{K} 1$ \\
\hline vp19 & RIMD 2210050 & Patient & Japan & Kobe & & 1972 & + & + & $\mathrm{O} 4: \mathrm{K} 12$ \\
\hline vp20 & RIMD 2210056 & Patient & Japan & Kobe & & 1972 & + & - & O4:K8 \\
\hline vp21 & RIMD 2210384 & Environment & Japan & Osaka & & 1976 & - & - & O4:KUT \\
\hline vp22 & RIMD 2210600 & Patient & Japan & Osaka & Kansai aq & 1996 & + & - & $\mathrm{O} 3: \mathrm{K} 5$ \\
\hline vp23 & RIMD 2210706 & Patient & Japan & Osaka & Osaka aq & 1993 & + & - & $\mathrm{O} 4: \mathrm{K} 12$ \\
\hline vp24 & RIMD 2210877 & Patient & Japan & Osaka & Osaka aq & 1992 & + & - & O1:KUT \\
\hline vp25 & RIMD 2210884 & Patient & Japan & Osaka & Osaka aq & 1992 & + & - & $\mathrm{O} 4: \mathrm{K} 8$ \\
\hline vp26 & RIMD 2210986 & Environment & Thailand & Bangkok & & 1994 & - & - & O1:KUT \\
\hline vp27 & RIMD 2211424 & Patient & Japan & Osaka & Osaka aq & 1989 & + & + & O6:K18 \\
\hline vp28 & RIMD 2211499 & Patient & Japan & Osaka & Osaka aq & 1994 & + & - & $\mathrm{O} 2: \mathrm{K} 3$ \\
\hline vp29 & RIMD 2211932 & Patient & Japan & Osaka & Osaka aq & 1990 & + & - & O8:KUT \\
\hline
\end{tabular}

aq, airport quarantine station. vp, Vibrio parahaemolyticus. RIMD, Research Institute of Microbial Diseases, Osaka University.

Table 2. PCR and sequencing primers

\begin{tabular}{cclr}
\hline Gene name & Primer name & \multicolumn{1}{c}{ Primer sequence $\left(5^{\prime} \rightarrow 3^{\prime}\right)$} & Binding primer position $(\mathrm{nt})$ \\
\hline Phosphoglucomutase $(\mathrm{pgm})$ & pgm_F & AAAGATACCCACGCGTTGTC & 253 \\
& pgm_R & GCTAAGAAATGGTTCGGGTTCAT & 977 \\
Recombination/repair protein $(r e c A)$ & recA_F & TCGGTAAAGGCTCTATCATG & 59 \\
& recA_R & TTAAAGCCTTGGCCATACAT & 803 \\
DNA gyrase beta subunit $($ gyr $B)$ & gyrB_F & GAAGGTGGTATTCAAGCG & 655 \\
& gyrB_R & AGAGTCACCCTCCACAATGTA & 1284
\end{tabular}

F, forward; R, reverse. The standard sequence used for primer design is the strain V. parahaemolyticus vp 2 (RIMD2210633).

haemolyticus and its potential effects in the whole genome of this bacterium. Homologous recombination may act as a cohesion force that purges genome wide diversity among conspecific strains ${ }^{4)}$. If this hypothesis is true, one should be able to detect homologous recombination among strains with highly related genomes. The aim of this study was to 
access the genetic similarity of a representative collection of clinical and environmental $V$. parahaemolyticus strains by means of amplified fragment length polymorphism (AFLP) and multilocus sequence analysis (MLSA) approaches. In addition, different types of recombination detection analyses were performed in order to check if recombination may have occurred between closely related $V$. parahaemolyticus strains.

\section{Materials and Methods}

\section{Bacterial strains}

The 29 representative strains used in this study represent well characterized strains from both clinical and environmental sources, including strains with different serotypes carrying thermostable directed hemolysin $(t d h)$ and thermostable related hemolysin (trh) genes (Table 1). Strains were grown on Trypton Soy Agar supplemented with $2 \% \mathrm{NaCl}$ at $27^{\circ} \mathrm{C}$ for $24 \mathrm{~h}$. DNA was extracted as described previously ${ }^{20)}$.

\section{AFLP analysis}

Template preparation, PCR, and electrophoresis were carried out essentially as described previously ${ }^{20}$. Band patterns of 50 to 536 base pairs, were transferred into BioNumerics 2.0 software (Applied Maths, St-Martens-Latem, Belgium) for numerical analysis. Clustering of the patterns was done using Dice and UPGMA ${ }^{19}$. For the comparison of the fragments, a band position tolerance value of $0.5 \%$ was allowed to compensate for the misalignment of homologous bands due to technical imperfections.

\section{Gene sequence analysis}

The primers and the respective amplified positions of the three genetic loci ( $r e c A, g y r B$, and $p g m$ ) examined in this study are listed in Table 2 . The amplification and sequencing of fragments of these genes were performed as described previously ${ }^{22)}$. The annealing temperature was $55^{\circ} \mathrm{C}$ for all three loci. Two runs were generated for each gene. Sequences were assembled with Kodon (Applied Biosystems, St-Martens-Latem, Belgium).

\section{Numerical analyses}

The sequences were aligned using ClustalX and phylogenetic trees were constructed by the neighbor-joining method (MEGA ver. 4.0 ${ }^{18)}$. The robustness of each topology was checked by 500 bootstrap replications. Trees were drawn by using MEGA version 4.0 $0^{12)}$.

Recombination was analyzed in each gene by using
SplitsTree version $4^{8)}$, the Phi statistical test ${ }^{2)}$, Sawyers ${ }^{17)}$, the Index of Association $(I a)^{13)}$, and Homoplasy ${ }^{14)}$ that are implemented in the software package START (http://pubmlst.org/software/analysis/start/ ${ }^{10)}$. The GC content and the ratio of mean synonymous substitutions per synonymous site/mean non-synonymous substitutions per non-synonymous site $(d s / d n)$ were also calculated using START. The gene sequences have been deposited in our own online database (http://www.taxvibrio.lncc.br/) and in GenBank under the accession nos. EF601726-EF601812.

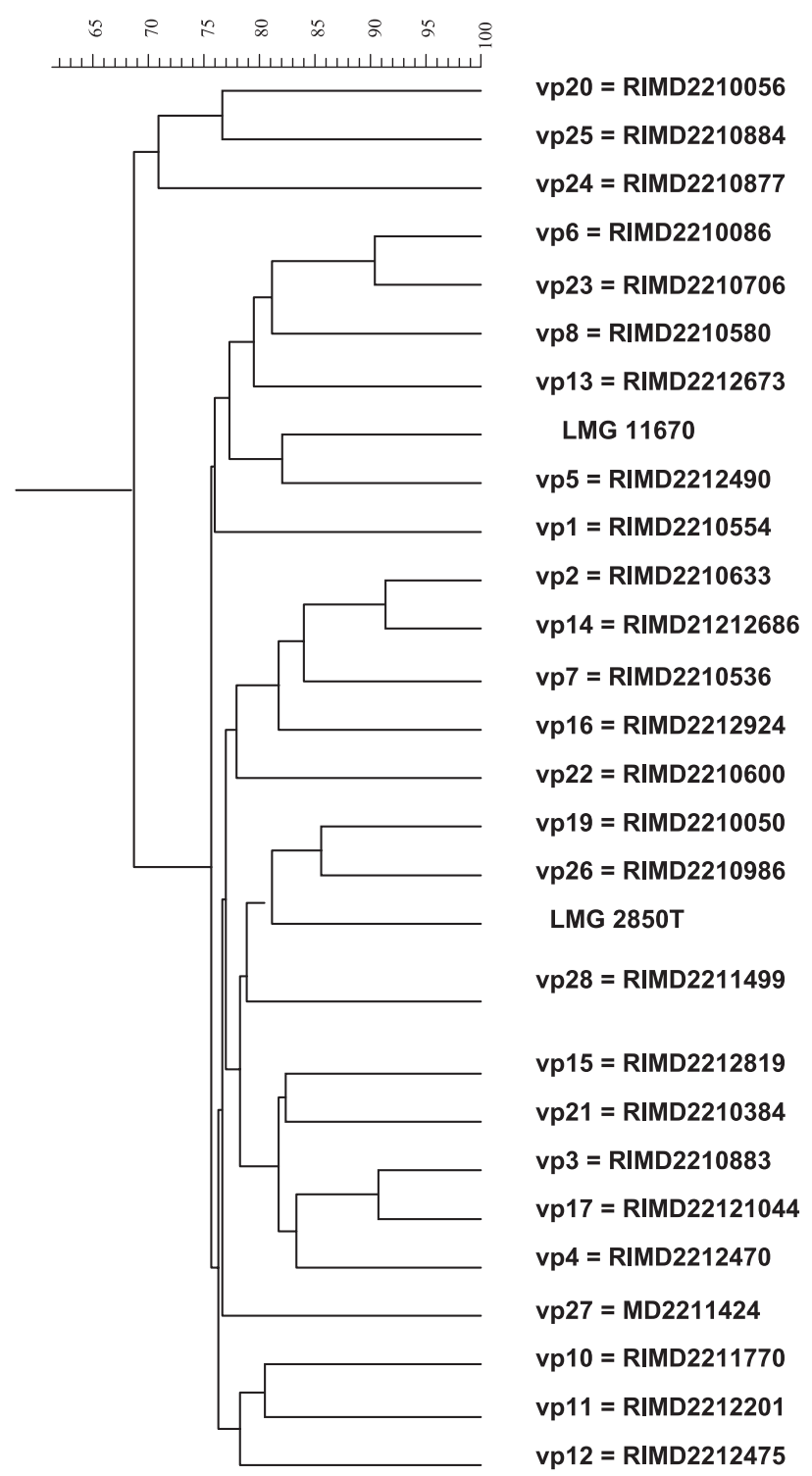

Fig. 1. Dendrogram based on the AFLP band patterns using the Dice band-based similarity and Ward dendrogram building algorithm. A band position of $0.5 \%$ was allowed. LMG $2850^{\mathrm{T}}$ and LMG 11670 originated from a diseased individual in Japan and Water of Yellow Sea (China), respectively. 


\section{Results and Discussion}

The AFLP band patterns consisted of around 90 fragments ranging from 75 to $500 \mathrm{bp}$ in size (Fig. 1). The highly heterogeneous strains vp20, vp24, and vp25 appeared at the outskirts of the cluster, but these strains clearly belong to the species $V$. parahaemolyticus because they share $>65 \%$ AFLP pattern similarity with LMG $2850^{\mathrm{T}}$. It is well known that AFLP pairwise similarity above $60 \%$ corresponds to DDH similarity above $70 \%$ which circumscribes the species level ${ }^{21,23)}$. Some pairs of strains had nearly identical AFLP patterns (i.e. $>90 \%$ similarity, equivalent to the level of reproducibility of this technique), e.g. vp6 and vp23, vp2 and vp14, vp3 and vp17. These pairs are indistinguishable by AFLP and, thus, they may be considered clonal. Strains vp6 and vp23 were isolated 25 years apart each other, yet they showed a remarkable similarity of genomes, including the presence of a $t d h$ gene. Possibly these strains have highly successful genomes in the environment. The pan- demic strains vp2, vp14, vp7, and vp16 had highly related genomes with more than $80 \%$ mutual similarity. These strains belong to serotypes associated with pandemics (O3:O6, O4:K68). The AFLP analysis revealed that both clinical and environmental isolates of $V$. parahaemolyticus share a common genetic background, suggesting that pandemic strains may arise from environmental strains via e.g. transduction or transformation ${ }^{13-15)}$. Hovewer, environmental strains did not possess $t d h$ and trh genes.

The grouping shown by the phylogenetic trees based on the gene sequences of $r e c A, g y r B$, and $p g m$ was in agreement with the AFLP dendrogram (Fig. 2). For instance, strains with nearly identical AFLP patterns always had identical gene sequences. However, the comparison of the three phylogenetic revealed that each gene possibly had a slightly different evolutionary story. According to $\operatorname{gyr} B$ gene sequences strain vp5 seems to be an earlier ancestor of the species from which diversification might have occurred. Two groups of highly related strains (i.e. vp16, vp3, vp17,

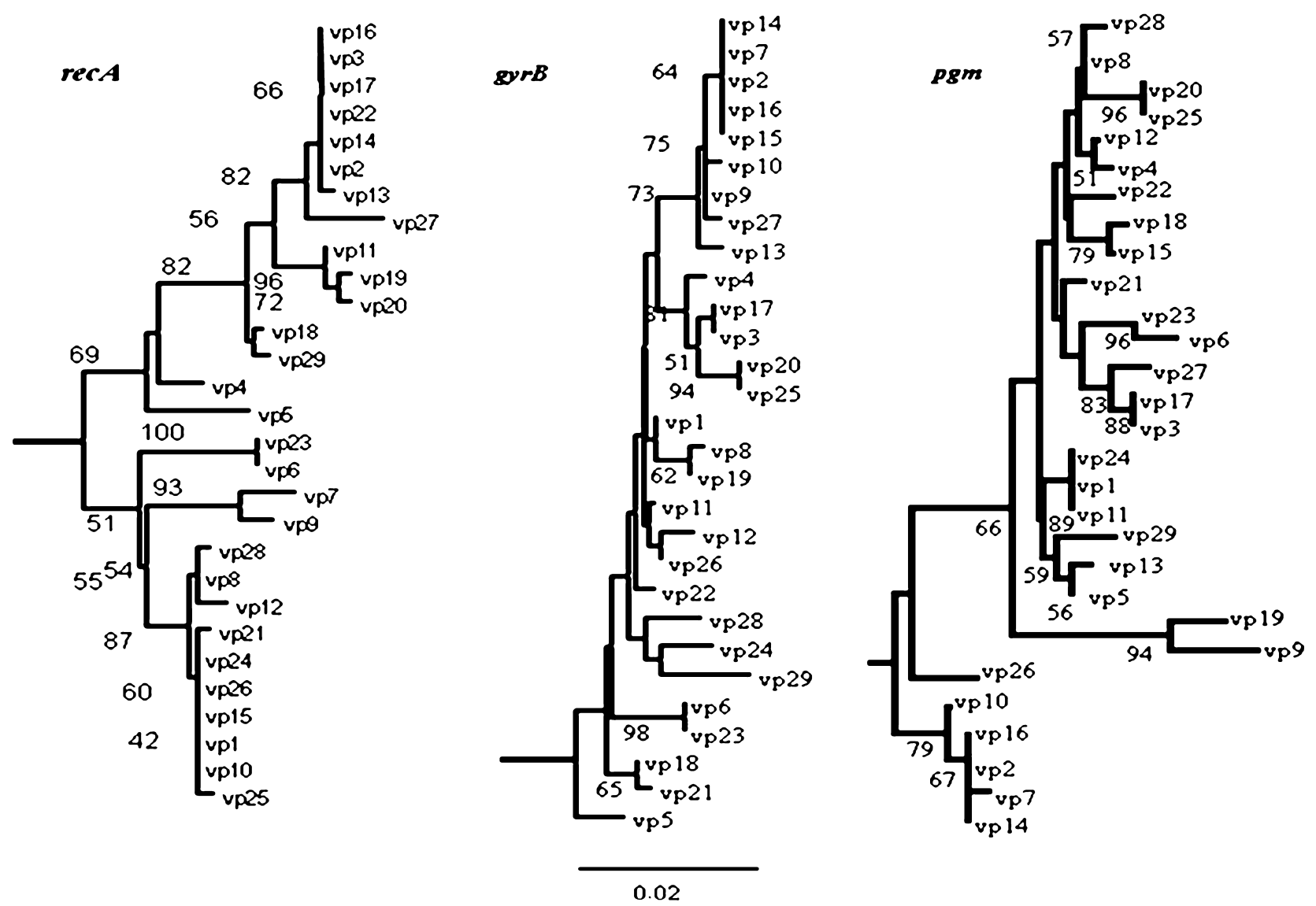

Fig. 2. Phylogenetic trees based on the Neigbour Joining method with the Kimura-Two parameter model and the fragments of the $r e c A$, $g y r B$, and $\mathrm{pgm}$ genes. Bootstrap values after 500 repetitions are shown. The scale bar is indicated ( $2 \%$ sequence difference). The trees were rooted with Photobacterium profundum SS9 gene sequences. 
Table 3. Summary of gene features

\begin{tabular}{lccccccr}
\hline \multicolumn{1}{c}{ Gene name } & Length (nt) & $\mathrm{dn} / \mathrm{ds}$ & $\begin{array}{c}\text { \% GC } \\
(\mathrm{Mean} \pm \mathrm{SD})\end{array}$ & No. alleles & $\begin{array}{c}\text { Recombination* } \\
\text { Phi }\end{array}$ & Sawyers & Homoplasy** \\
\hline RecA protein (recA) & 633 & 0.002 & $46.01 \pm 0.43$ & 20 & $<0.001$ & 0.00 & 0.004 \\
DNA gyrase, subunit B (gyrB) & 510 & 0.000 & $48.80 \pm 0.26$ & 22 & 0.1 & 0.02 & $<0.001$ \\
Phosphoglucomutase (pgm) & 588 & 0.101 & $46.13 \pm 0.20$ & 24 & $<0.001$ & $<0.001$ & $<0.001$ \\
\hline
\end{tabular}

Phi recombination statistics (Bruen et al., 2006). * P value, statistical significance. ** The ratio of true homoplasies/expected homoplasies if clonal for $g y r B$, recA, and $p g m$ were $14 / 2.9,151 / 6.1$, and $14 / 3.7$.

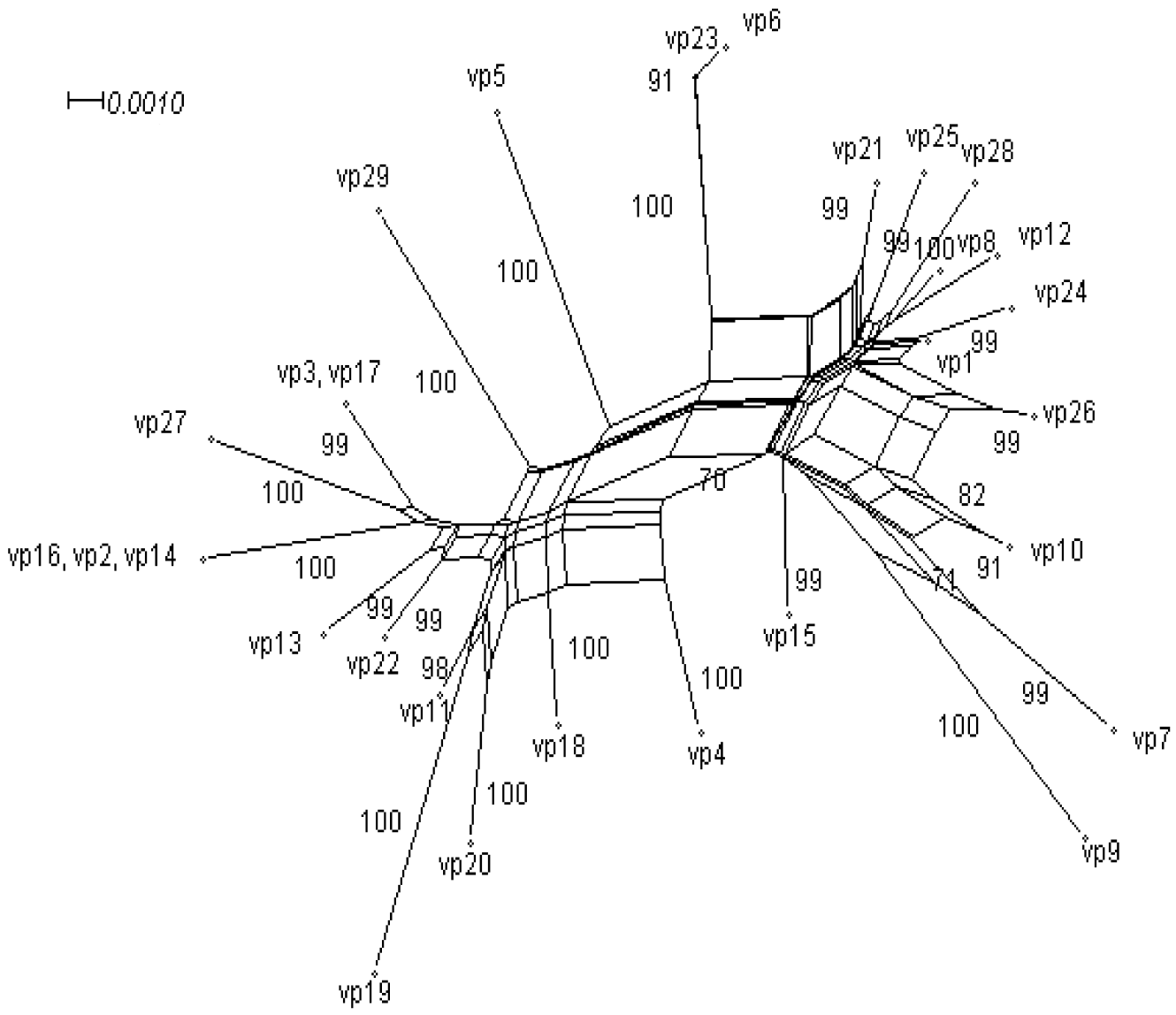

Fig. 3. Splits decomposition tree based on the concatenated gene sequences of $r e c A$, gyrB, and pgm (1731 nt). Bootstrap values after 500 repetitions are shown. The scale bar is indicated $(0.1 \%$ sequence difference). Fit=91.5; fit of the data into the model of reticulate evolution.

vp22, vp14, vp2, and vp13; vp28, vp8, vp12, vp21, vp24, vp26, vp15, vp1, vp10, and vp25) on the basis of $r e c A$ gene sequences appeared scattered in the other two trees. Recombination has been deemed one of the causes of incongruence in the topology of phylogenetic trees ${ }^{3,6)}$.

The three genetic loci examined in this study showed low $d n / d s$ typical of housekeeping phylogeneticaly informative genes (Table 3). The splits decomposition analysis and the Phi test based on the concatenated sequences of the three genes did point out to a reticulate evolution possibly due to recombination (Table 3, Figure 3). The linkage disequilibrium was evaluated using the allelic profiles of each strain (Table 4$)$. The observed value of variance $(0.147)$ was higher than the expected variance $(0.099)$ if the strains were experiencing free recombination with an Index of association $(I a=0.482)$. This analysis suggested that the population was in linkage disequilibrium, but this does not preclude that recombination between strains did indeed take place ${ }^{3)}$. 
Table 4. Summary of allelic profiles

\begin{tabular}{|c|c|c|c|}
\hline Strain & gyrB & pgm & recA \\
\hline vp1 & 6 & 16 & 12 \\
\hline vp2 & 7 & 22 & 7 \\
\hline vp3 & 11 & 13 & 6 \\
\hline vp4 & 12 & 10 & 10 \\
\hline vp5 & 2 & 19 & 11 \\
\hline vp6 & 1 & 12 & 20 \\
\hline vp7 & 7 & 21 & 18 \\
\hline vp8 & 5 & 8 & 16 \\
\hline vp9 & 8 & 2 & 19 \\
\hline vp10 & 9 & 23 & 12 \\
\hline vp11 & 4 & 17 & 5 \\
\hline vp12 & 3 & 9 & 17 \\
\hline vp13 & 10 & 18 & 8 \\
\hline vp14 & 7 & 22 & 6 \\
\hline vp15 & 7 & 4 & 12 \\
\hline vp16 & 7 & 22 & 6 \\
\hline vp17 & 11 & 13 & 6 \\
\hline vp18 & 13 & 3 & 1 \\
\hline vp19 & 14 & 1 & 3 \\
\hline vp20 & 15 & 8 & 4 \\
\hline vp21 & 16 & 15 & 14 \\
\hline vp22 & 17 & 5 & 6 \\
\hline vp23 & 1 & 11 & 20 \\
\hline vp24 & 18 & 16 & 12 \\
\hline vp25 & 15 & 8 & 13 \\
\hline vp26 & 19 & 24 & 12 \\
\hline vp27 & 20 & 14 & 9 \\
\hline vp28 & 21 & 6 & 15 \\
\hline vp29 & 22 & 20 & 2 \\
\hline
\end{tabular}

The $I a$ alone is not a good predictor of recombination if the collection of strains is small and/or biased through clinical isolates where the clonal spread of a successful strain is very common. Ideally different recombination detection tests should be applied in order to confidently find recombination events. In this study, the tests Phi, Sawyers, and Homoplasy did point to recombination (Table 3).

The Phi recombination test takes polymorphic sites in an alignment with opposing incongruent phylogenetic signals as breakpoints of recombination, while the Homoplasy test points out that excess Homoplasy (that is, shared similarity in different branches of a phylogenetic tree not inherited directly from an ancestor) is likely to have occurred due to recombination. The most parcimonious explanation for finding identical substitutions in different branches of a tree is recombination. Several events of mutation would be necessary to explain Homoplasies. According to Sawyer
(1989) ${ }^{17}$, a gene conversion event will increase the value of the sum of the squares of condensed fragments (SSCF) because it results in an identical region within two sequences and may produce an unusually long fragment.

In this study we analyzed the gene sequences of $V$. parahaemolyticus by means of several recombination detection methods. Overall, our analysis points out that homologous recombination between conspecific strains of Vibrio parahaemolyticus is an important force possibly purging diversification of this species. Work is under way to unravel the impact of genome-wide recombination in the evolution of Vibrio parahaemolyticus genomes. Recombination rates may well vary in different portions of the genomes, with hypervariable (e.g. the superintegron region) and conserved regions (e.g. the oriC regions), playing distinct roles in the dynamics of vibrios genomes and their biology.

\section{Acknowledgements}

F. L. T. acknowledges grants of CNPq, IFS, and FUJB. F. L. T. acknowledges a JSPS short-term visit fellowship (Japan). This work was supported by a JSPS short-term visit fellowship (Japan) and Grants-in-Aid for Scientific Research on Priority Areas and Scientific Research from the Ministry of Education, Culture, Sports, Science, and Technology of Japan. The BCCM ${ }^{\mathrm{TM}} / \mathrm{LMG}$ Bacteria Collection is supported by the Prime Minister's Services, Federal Office for Scientific, Technical and Cultural Affairs, Belgium.

\section{References}

1) Alsina, M., and A.R. Blanch. 1994. A set of keys for biochemical identification of environmental Vibrio species. J. Appl. Bacteriol. 76:79-85.

2) Bruen, T.C., H. Philippe, and D. Bryant. 2006. A simple and robust statistical test for detecting the presence of recombination. Genetics. 172:2665-2681.

3) Feil, E.J. 2004. Small change: keeping pace with microevolution. Nat. Rev. Microbiol. 2:483-495.

4) Gevers, D., F.M. Cohan, J.G. Lawrence, B.G. Spratt, T. Coenye, E.J. Feil, E. Stackebrandt, Y. Van de Peer, P. Vandamme, F.L. Thompson, and J. Swings. 2005. Opinion: Re-evaluating prokaryotic species. Nat. Rev. Microbiol. 3:733-739.

5) Gomez-Gil, B., S. Soto-Rodríguez, A. García-Gasca, A. Roque, R. Vazquez-Juarez, F.L. Thompson, and J. Swings. 2004. Molecular identification of Vibrio harveyi-related isolates associated with diseased aquatic organisms. Microbiology 150:1769-1777.

6) Hanage, W.P., C. Fraser, and B.G. Spratt. 2005. Fuzzy species among recombinogenic bacteria. BMC Biol. 7:3-6.

7) Henke, J., and B. Bassler. 2004. Quorum sensing regulates type III secretion in Vibrio harveyi and Vibrio parahaemolyticus. J. Bacteriol. 186:3794-3805.

8) Huson, D.H. 1998. SplitsTree: analyzing and visualizing evolu- 
tionary data. Bioinformatics 14:68-73.

9) Iida, T., K.S. Park, O. Suthienkul, J. Kozawa, Y. Yamaichi, K. Yamamoto, and T. Honda. 1998. Close proximity of the $t d h, t r h$, and ure genes on the chromosome of Vibrio parahaemolyticus. Microbiology 144:2517-2523.

10) Jolley, K.A., E.J. Feil, M.S. Chan, and M.C. Maiden. 2001. Sequence type analysis and recombinational tests (START). Bioinformatics 17:1230-1231.

11) Kimura, B. 2006. Recent advances in the study of the genotypic diversity and ecology of Listeria monocytogenes. Microbes Environ. 21:69-77.

12) Kumar, S., K. Tamura, and M. Nei. 2004. MEGA3: Integrated software for Molecular Evolutionary Genetics Analysis and sequence alignment. Brief Bioinform. 5:150-163.

13) Maynard-Smith, J., N.H. Smith, M. O'Rourke, and B.G. Spratt. 1993. How clonal are bacteria? Proc. Natl. Acad. Sci. USA 90:4384-4388.

14) Maynard-Smith, J., and N.H. Smith. 1998. Detecting recombination from gene trees. Mol. Biol. Evol. 15:590-599.

15) Meibom, K.L., M. Blokesch, N.A. Dolganov, C.Y. Wu, and G.K. Schoolnik. 2005. Chitin induces natural competence in Vibrio cholerae. Science 310:1824-1827.

16) Nasu, H., T. Iida, T. Sugahara, Y. Yamaichi, K.S. Park, K. Yokoyama, K. Makino, H. Shinagawa, and T. Honda. 2000. A filamentous phage associated with recent pandemic Vibrio parahaemolyticus O3:K6 strains. J. Clin. Microbiol. 38:2156-2161.

17) Sawyer, S. 1989. Statistical tests for detecting gene conversion. Mol. Biol. Evol. 6:526-538.

18) Saitou, N., and M. Nei. 1987. The neighbor-joining method: a new method for reconstructing phylogenetic trees. Mol. Biol.
Evol. 4:406-425.

19) Sneath, P.H.A., and R.R. Sokal. 1973. Numerical Taxonomy. The principles and practice of numerical classification. Freeman. San Francisco.

20) Thompson, F.L., B. Hoste, K. Vandemeulebroecke, and J. Swings. 2001. Genomic diversity amongst Vibrio isolates from different sources determined by fluorescent amplified fragment length polymorphism. Syst. Appl. Microbiol. 24:520-538.

21) Thompson, F.L., T. Iida, and J. Swings. 2004. Biodiversity of vibrios. Microbiol. Mol. Biol. Rev. 68:403-431.

22) Thompson, F.L., D. Gevers, C.C. Thompson, P. Dawyndt, S. Naser, B. Hoste, C.B. Munn, and J. Swings. 2005. Phylogeny and molecular identification of vibrios on the basis of multilocus sequence analysis. Appl. Environ. Microbiol. 71:5107-5115.

23) Thompson, F.L., and J. Swings. 2006. Taxonomy of vibrios, pp. 29-43. In F.L. Thompson, B. Austin, and J. Swings (ed.), The Biology of Vibrios, ASM Press, Washington D.C.

24) Thompson, F.L., B. Gomez-Gil, A.T.R. Vasconcelos, and T. Sawabe. 2007. Multilocus sequence analysis reveals that Vibrio harveyi and $V$. campbellii form distinct species. Appl. Environ. Microbiol. 73:4279-4285.

25) Wong, H.C., K.T. Lu, T.M. Pan, C.L. Lee, and D.Y. Shih. 1996 Subspecies typing of Vibrio parahaemolyticus by pulsed-field gel electrophoresis. J. Clin. Microbiol. 34:1535-1539.

26) Wong, H.C., S.H. Liu, C.S. Chiou, M. Nishibuchi, B.K. Lee, O. Suthienkul, G.B. Nair, C.A. Kaysner, and H. Taniguchi. 2007. A pulsed-field gel electrophoresis typing scheme for Vibrio parahaemolyticus isolates from fifteen countries. Int. J. Food Microbiol. 114:280-287. 